TRANSACTIONS OF THE

AMERICAN MATHEMATICAL SOCIETY

Volume 358, Number 4 , Pages 1605-1617

S 0002-9947(05)03764-5

Article electronically published on June 21, 2005

\title{
ON THE COHEN-MACAULAY PROPERTY OF MULTIPLICATIVE INVARIANTS
}

\author{
MARTIN LORENZ
}

\begin{abstract}
We investigate the Cohen-Macaulay property for rings of invariants under multiplicative actions of a finite group $\mathcal{G}$. By definition, these are $\mathcal{G}$-actions on Laurent polynomial algebras $\mathbb{k}\left[x_{1}^{ \pm 1}, \ldots, x_{n}^{ \pm 1}\right]$ that stabilize the multiplicative group consisting of all monomials in the variables $x_{i}$. For the most part, we concentrate on the case where the base ring $\mathbb{k}$ is $\mathbb{Z}$. Our main result states that if $\mathcal{G}$ acts non-trivially and the invariant ring $\mathbb{Z}\left[x_{1}^{ \pm 1}, \ldots, x_{n}^{ \pm 1}\right]^{\mathcal{G}}$ is Cohen-Macaulay, then the abelianized isotropy groups $\mathcal{G}_{m}^{\text {ab }}$ of all monomials $m$ are generated by the bireflections in $\mathcal{G}_{m}$ and at least one $\mathcal{G}_{m}^{\text {ab }}$ is non-trivial. As an application, we prove the multiplicative version of Kemper's 3-copies conjecture.
\end{abstract}

\section{INTRODUCTION}

This article is a sequel to $[\mathrm{LPk}$. Unlike in $[\mathrm{LPk}$, however, our focus will be specifically on multiplicative invariants. In detail, let $L \cong \mathbb{Z}^{n}$ denote a lattice on which a finite group $\mathcal{G}$ acts by automorphisms. The $\mathcal{G}$-action on $L$ extends uniquely to an action by $\mathbb{k}$-algebra automorphisms on the group algebra $\mathbb{k}[L] \cong$ $\mathbb{k}\left[x_{1}^{ \pm 1}, \ldots, x_{n}^{ \pm 1}\right]$ over any commutative base ring $\mathbb{k}$. We are interested in the question of when the subalgebra $\mathbb{k}[L]^{\mathcal{G}}$ consisting of all $\mathcal{G}$-invariant elements of $\mathbb{k}[L]$ has the Cohen-Macaulay property. The reader is assumed to have some familiarity with Cohen-Macaulay rings; a good reference on this subject is $[\mathrm{BH}]$.

It is a standard fact that $\mathbb{k}[L]$ is Cohen-Macaulay precisely if $\mathbb{k}$ is. On the other hand, while $\mathbb{k}[L]^{\mathcal{G}}$ can only be Cohen-Macaulay when $\mathbb{k}$ is so, the latter condition is far from sufficient, and rather stringent additional conditions on the action of $\mathcal{G}$ on $L$ are required to ensure that $\mathbb{k}[L]^{\mathcal{G}}$ is Cohen-Macaulay. Remarkably, the question of whether or not $\mathbb{k}[L]^{\mathcal{G}}$ is Cohen-Macaulay, for any given base ring $\mathbb{k}$, depends only on the rational isomorphism class of the lattice $L$, that is, the isomorphism class of $L \otimes_{\mathbb{Z}} \mathbb{Q}$ as $\mathbb{Q}[\mathcal{G}]$-module; see Proposition 3.4 below. This is in striking contrast with most other ring theoretic properties of $\mathbb{k}[L]^{\mathcal{G}}$ (e.g., regularity, structure of the class group) which tend to be sensitive to the $\mathbb{Z}$-type of $L$. For an overview, see $\mathrm{L}_{1}$.

We will largely concentrate on the case where the base ring $\mathbb{k}$ is $\mathbb{Z}$. This is justified in part by the fact that if $\mathbb{Z}[L]^{\mathcal{G}}$ is Cohen-Macaulay, then likewise is $\mathbb{k}[L]^{\mathcal{G}}$

Received by the editors December 15, 2003 and, in revised form, May 26, 2004.

2000 Mathematics Subject Classification. Primary 13A50, 16W22, 13C14, 13 H10.

Key words and phrases. Finite group action, ring of invariants, multiplicative invariant theory, height, depth, Cohen-Macaulay ring, group cohomology, generalized reflections, bireflections, integral representation, binary icosahedral group.

This research was supported in part by NSF grant DMS-9988756.

(C)2005 American Mathematical Society Reverts to public domain 28 years from publication 
for any Cohen-Macaulay base ring $\mathbb{k}$ (Lemma 3.2 ). Assuming $\mathbb{Z}[L]^{\mathcal{G}}$ to be CohenMacaulay, we aim to derive group theoretical consequences for the isotropy groups $\mathcal{G}_{m}=\{g \in \mathcal{G} \mid g(m)=m\}$ with $m \in L$. An element $g \in \mathcal{G}$ will be called a $k$ reflection on $L$ if the sublattice $[g, L]=\{g(m)-m \mid m \in L\}$ of $L$ has rank at most $k$ or, equivalently, if the $g$-fixed points of the $\mathbb{Q}$-space $L \otimes_{\mathbb{Z}} \mathbb{Q}$ have codimension at most $k$. As usual, $k$-reflections with $k=1$ and $k=2$ will be referred to as reflections and bireflections. For any subgroup $\mathcal{H} \leq \mathcal{G}$, we let $\mathcal{H}^{(2)}$ denote the subgroup generated by the elements of $\mathcal{H}$ that act as bireflections on $L$. Our main result now reads as follows.

Theorem. Assume that $\mathbb{Z}[L]^{\mathcal{G}}$ is Cohen-Macaulay. Then $\mathcal{G}_{m} / \mathcal{G}_{m}^{(2)}$ is a perfect group (i.e., equal to its commutator subgroup) for all $m \in L$. If $\mathcal{G}$ acts non-trivially on $L$, then some $\mathcal{G}_{m}$ is non-perfect.

It would be interesting to determine if the conclusion of the theorem can be strengthened to the effect that all isotropy groups $\mathcal{G}_{m}$ are in fact generated by bireflections on $L$. I do not know if, for the latter to occur, it is sufficient that $\mathcal{G}$ is generated by bireflections. The corresponding fact for reflection groups is known to be true: if $\mathcal{G}$ is generated by reflections on $L$ (or, equivalently, on $L \otimes_{\mathbb{Z}} \mathbb{Q}$ ), then so are all isotropy groups $\mathcal{G}_{m}$; see [St, Theorem 1.5] or [Bou 1 , Exercise 8(a) on p. 139].

There is essentially a complete classification of finite linear groups generated by bireflections. In arbitrary characteristic, this is due to Guralnick and Saxl GuS]; for the case of characteristic zero, see Huffman and Wales [HuW]. Bireflection groups have been of interest in connection with the problem of determining all finite linear groups whose algebra of polynomial invariants is a complete intersection. Specifically, suppose that $\mathcal{G} \leq \mathrm{GL}(V)$ for some finite-dimensional vector space $V$ and let $\mathcal{O}(V)=\mathrm{S}\left(V^{*}\right)$ denote the algebra of polynomial functions on $V$. It was shown by Kac and Watanabe $\left[\mathrm{KW}\right.$ and independently by Gordeev $\mathrm{G}_{1}$ that if the algebra $\mathcal{O}(V)^{\mathcal{G}}$ of all $\mathcal{G}$-invariant polynomial functions is a complete intersection, then $\mathcal{G}$ is generated by bireflections on $V$. The classification of all groups $\mathcal{G}$ so that $\mathcal{O}(V)^{\mathcal{G}}$ is a complete intersection has been achieved by Gordeev $\mathrm{G}_{2}$ and by Nakajima $[\mathrm{N}$.

The last assertion of the above Theorem implies in particular that if $\mathbb{Z}[L]^{\mathcal{G}}$ is Cohen-Macaulay and $\mathcal{G}$ acts non-trivially on $L$, then some element of $\mathcal{G}$ acts as a non-trivial bireflection on $L$. Hence we obtain the following multiplicative version of Kemper's 3-copies conjecture:

Corollary. If $\mathcal{G}$ acts non-trivially on $L$ and $r \geq 3$, then $\mathbb{Z}\left[L^{\oplus r}\right]^{\mathcal{G}}$ is not Cohen-Macaulay.

The 3-copies conjecture was formulated by Kemper [ $\mathrm{K}_{1}$, Vermutung 3.12] in the context of polynomial invariants. Using the above notation, the original conjecture states that if $1 \neq \mathcal{G} \leq \mathrm{GL}(V)$ and the characteristic of the base field of $V$ divides the order of $\mathcal{G}$ ("modular case"), then the invariant algebra $\mathcal{O}\left(V^{\oplus r}\right)^{\mathcal{G}}$ will not be Cohen-Macaulay for any $r \geq 3$. This is still open. The main factors contributing to our success in the multiplicative case are the following:

- Multiplicative actions are permutation actions: $\mathcal{G}$ permutes the $\mathbb{k}$-basis of $\mathbb{k}[L]$ consisting of all "monomials", corresponding to the elements of the lattice $L$. Consequently, the cohomology $H^{*}(\mathcal{G}, \mathbb{k}[L])$ is simply the direct sum of the various $H^{*}\left(\mathcal{G}_{m}, \mathbb{k}\right)$ with $m$ running over a transversal for the $\mathcal{G}$-orbits in $L$. 
- Up to conjugacy, there are only finitely many finite subgroups of $\mathrm{GL}_{n}(\mathbb{Z})$, and these groups are explicitly known for small $n$. A crucial observation for our purposes is the following: if $\mathcal{G}$ is a non-trivial finite perfect subgroup of $\mathrm{GL}_{n}(\mathbb{Z})$ such that no $1 \neq g \in \mathcal{G}$ has eigenvalue 1 , then $\mathcal{G}$ is isomorphic to the binary icosahedral group and $n \geq 8$; see Lemma 2.3 below.

A brief outline of the contents of the this article is as follows. The short preliminary Section 1 is devoted to general actions of a finite group $\mathcal{G}$ on a commutative ring $R$. This material relies rather heavily on $[\mathrm{LPk}$. We liberate a technical result from $\mathrm{LPk}$ from any a priori hypotheses on the characteristic; the new version (Proposition 1.4) states that if $R$ and $R^{\mathcal{G}}$ are both Cohen-Macaulay and $H^{i}(\mathcal{G}, R)=0$ for $0<i<k$, then $H^{k}(\mathcal{G}, R)$ is detected by $k+1$-reflections. Section 2 then specializes to the case of multiplicative actions. We assemble the main tools required for the proof of the Theorem, which is presented in Section 3. The article concludes with a brief discussion of possible avenues for further investigation and some examples.

\section{Finite GROUP ACTIONS ON RINGS}

1.1. In this section, $R$ will be a commutative ring on which a finite group $\mathcal{G}$ acts by ring automorphisms $r \mapsto g(r)(r \in R, g \in \mathcal{G})$. The subring of $\mathcal{G}$-invariant elements of $R$ will be denoted by $R^{\mathcal{G}}$.

1.2. Generalized reflections. Following [GK], we will say an element $g \in \mathcal{G}$ acts as a $k$-reflection on $R$ if $g$ belongs to the inertia group

$$
I_{\mathcal{G}}(\mathfrak{P})=\{g \in \mathcal{G} \mid g(r)-r \in \mathfrak{P} \forall r \in R\}
$$

of some prime ideal $\mathfrak{P} \in \operatorname{Spec} R$ with height $\mathfrak{P} \leq k$. The cases $k=1$ and $k=2$ will be referred to as reflections and bireflections, respectively. Define the ideal $I_{R}(g)$ of $R$ by

$$
I_{R}(g)=\sum_{r \in R}(g(r)-r) R .
$$

Evidently, $\mathfrak{P} \supseteq I_{R}(g)$ is equivalent to $g \in I_{\mathcal{G}}(\mathfrak{P})$. Thus:

$g$ is a $k$-reflection on $R$ if and only if height $I_{R}(g) \leq k$.

For each subgroup $\mathcal{H} \leq \mathcal{G}$, we put

$$
I_{R}(\mathcal{H})=\sum_{g \in \mathcal{H}} I_{R}(g)
$$

It suffices to let $g$ run over a set of generators of the group $\mathcal{H}$ in this sum.

1.3. A height estimate. The cohomology $H^{*}(\mathcal{G}, R)=\bigoplus_{n \geq 0} H^{n}(\mathcal{G}, R)$ has a canonical $R^{\mathcal{G}}$-module structure: for each $r \in R^{\mathcal{G}}$, the map $\rho: R \rightarrow R, s \mapsto r s$, is $\mathcal{G}$ equivariant and hence it induces a map on cohomology $\rho_{*}: H^{*}(\mathcal{G}, R) \rightarrow H^{*}(\mathcal{G}, R)$. The element $r$ acts on $H^{*}(\mathcal{G}, R)$ via $\rho_{*}$. Let $\operatorname{res}_{\mathcal{H}}^{\mathcal{G}}: H^{*}(\mathcal{G}, R) \rightarrow H^{*}(\mathcal{H}, R)$ denote the restriction map.

The following lemma extends $[\mathrm{LPk}$, Proposition 1.4].

Lemma. For any $x \in H^{*}(\mathcal{G}, R)$,

$$
\text { height } \operatorname{ann}_{R^{\mathcal{G}}}(x) \geq \inf \left\{\text { height } I_{R}(\mathcal{H}) \mid \mathcal{H} \leq \mathcal{G}, \operatorname{res}_{\mathcal{H}}^{\mathcal{G}}(x) \neq 0\right\} .
$$


Proof. Put $\mathfrak{X}=\left\{\mathcal{H} \leq \mathcal{G} \mid \operatorname{res}_{\mathcal{H}}^{\mathcal{G}}(x)=0\right\}$. For each $\mathcal{H} \leq \mathcal{G}$, let $R_{\mathcal{H}}^{\mathcal{G}}$ denote the image of the relative trace map $R^{\mathcal{H}} \rightarrow R^{\mathcal{G}}, r \mapsto \sum_{g} g(r)$, where $g$ runs over a transversal for the cosets $g \mathcal{H}$ of $\mathcal{H}$ in $\mathcal{G}$. By [LPk, Lemma 1.3],

$$
R_{\mathcal{H}}^{\mathcal{G}} \subseteq \operatorname{ann}_{R^{\mathcal{G}}}(x) \text { for all } \mathcal{H} \in \mathfrak{X} .
$$

To prove the lemma, we may assume that $\operatorname{ann}_{R^{\mathcal{G}}}(x)$ is a proper ideal of $R^{\mathcal{G}}$; for, otherwise height $\operatorname{ann}_{R^{\mathcal{G}}}(x)=\infty$. Choose a prime ideal $\mathfrak{p}$ of $R^{\mathcal{G}}$ with $\mathfrak{p} \supseteq \operatorname{ann}_{R^{\mathcal{G}}}(x)$ and height $\mathfrak{p}=$ height $\operatorname{ann}_{R^{\mathcal{G}}}(x)$. If $\mathfrak{P}$ is a prime of $R$ that lies over $\mathfrak{p}$, then

$$
R_{\mathcal{H}}^{\mathcal{G}} \subseteq \mathfrak{P} \text { for all } \mathcal{H} \in \mathfrak{X}
$$

and height $\mathfrak{P}=$ height $\mathfrak{p}$. By $[\mathrm{LPk}$, Lemma 1.1], the above inclusion implies that

$$
\left[I_{\mathcal{G}}(\mathfrak{P}): I_{\mathcal{H}}(\mathfrak{P})\right] \in \mathfrak{P} \quad \text { for all } \mathcal{H} \in \mathfrak{X} .
$$

Put $p=\operatorname{char} R / \mathfrak{P}$ and let $\mathcal{P} \leq I_{\mathcal{G}}(\mathfrak{P})$ be a Sylow $p$-subgroup of $I_{\mathcal{G}}(\mathfrak{P})$ (so $\mathcal{P}=1$ if $p=0)$. Then $I_{R}(\mathcal{P}) \subseteq \mathfrak{P}$ and $\left[I_{\mathcal{G}}(\mathfrak{P}): \mathcal{P}\right] \notin \mathfrak{P}$. Hence, $\mathcal{P} \notin \mathfrak{X}$ and height $I_{R}(\mathcal{P}) \leq$ height $\mathfrak{P}=$ height $\operatorname{ann}_{R^{\mathcal{G}}}(x)$. This proves the lemma.

We remark that the lemma and its proof carry over verbatim to the more general situation where $H^{*}(\mathcal{G}, R)$ is replaced by $H^{*}(\mathcal{G}, M)$, where $M$ is some module over the skew group ring of $\mathcal{G}$ over $R$; cf. [LPk]. However, we will not be concerned with this generalization here.

1.4. A necessary condition. In this section, we assume that $R$ is noetherian as an $R^{\mathcal{G}}$-module. This assumption is satisfied whenever $R$ is an affine algebra over some noetherian subring $\mathbb{k} \subseteq R^{\mathcal{G}}$; see $\left[\mathrm{Bou}_{2}\right.$, Théorème 2 on p. 33]. Put

$$
\mathfrak{X}_{k}=\left\{\mathcal{H} \leq \mathcal{G} \mid \text { height } I_{R}(\mathcal{H}) \leq k\right\} .
$$

Note that each $\mathcal{H} \in \mathfrak{X}_{k}$ consists of $k$-reflections on $R$. The following proposition is a characteristic-free version of [LPk, Proposition 4.1].

Proposition. Assume that $R$ and $R^{\mathcal{G}}$ are Cohen-Macaulay. If $H^{i}(\mathcal{G}, R)=0$ for $0<i<k$, then the restriction map

$$
\operatorname{res}_{\mathfrak{X}_{k+1}}^{\mathcal{G}}: H^{k}(\mathcal{G}, R) \rightarrow \prod_{\mathcal{H} \in \mathfrak{X}_{k+1}} H^{k}(\mathcal{H}, R)
$$

is injective.

Proof. We may assume that $H^{k}(\mathcal{G}, R) \neq 0$. Let $x \in H^{k}(\mathcal{G}, R)$ be non-zero and put $\mathfrak{a}=\operatorname{ann}_{R^{\mathcal{G}}}(x)$. By [LPk, Proposition 3.3], depth $\mathfrak{a} \leq k+1$. Since $R^{\mathcal{G}}$ is Cohen-Macaulay, depth $\mathfrak{a}=$ height $\mathfrak{a}$. Thus, Lemma 1.3 implies that $k+1 \geq$ height $I_{R}(\mathcal{H})$ for some $\mathcal{H} \leq \mathcal{G}$ with $\operatorname{res}_{\mathcal{H}}^{\mathcal{G}}(x) \neq 0$. The Proposition follows.

Note that the vanishing hypothesis on $H^{i}(\mathcal{G}, R)$ is vacuous for $k=1$. Thus, $H^{1}(\mathcal{G}, R)$ is detected by bireflections whenever $R$ and $R^{\mathcal{G}}$ are both Cohen-Macaulay.

\section{Multiplicative actions}

2.1. For the remainder of this article, $L$ will denote a lattice on which the finite group $\mathcal{G}$ acts by automorphisms $m \mapsto g(m)(m \in L, g \in \mathcal{G})$. The group algebra of $L$ over some commutative base ring $\mathbb{k}$ will be denoted by $\mathbb{k}[L]$. We will use additive notation in $L$. The $\mathbb{k}$-basis element of $\mathbb{k}[L]$ corresponding to the lattice element $m \in L$ will be written as

$$
\mathbf{x}^{m}
$$


so $\mathbf{x}^{0}=1, \mathbf{x}^{m+m^{\prime}}=\mathbf{x}^{m} \mathbf{x}^{m^{\prime}}$, and $\mathbf{x}^{-m}=\left(\mathbf{x}^{m}\right)^{-1}$. The action of $\mathcal{G}$ on $L$ extends uniquely to an action by $\mathbb{k}$-algebra automorphisms on $\mathbb{k}[L]$ :

$$
g\left(\sum_{m \in L} k_{m} \mathbf{x}^{m}\right)=\sum_{m \in L} k_{m} \mathbf{x}^{g(m)} .
$$

The invariant algebra $\mathbb{k}[L]^{\mathcal{G}}$ is a free $\mathbb{k}$-module: a $\mathbb{k}$-basis is given by the $\mathcal{G}$-orbit sums $\sigma(m)=\sum_{m^{\prime} \in \mathcal{G}(m)} \mathbf{x}^{m^{\prime}}$, where $\mathcal{G}(m)$ denotes the $\mathcal{G}$-orbit of $m \in L$. Since all orbit sums are defined over $\mathbb{Z}$, we have

$$
\mathbb{k}[L]^{\mathcal{G}}=\mathbb{k} \otimes_{\mathbb{Z}} \mathbb{Z}[L]^{\mathcal{G}} .
$$

2.2. Let $\mathcal{H}$ be a subgroup of $\mathcal{G}$. We compute the height of the ideal $I_{\mathbb{k}[L]}(\mathcal{H})$ from $\$ 1.2$ Let

$$
L^{\mathcal{H}}=\{m \in L \mid g(m)=m \text { for all } g \in \mathcal{H}\}
$$

denote the lattice of $\mathcal{H}$-invariants in $L$ and define the sublattice $[\mathcal{H}, L]$ of $L$ by

$$
[\mathcal{H}, L]=\sum_{g \in \mathcal{H}}[g, L],
$$

where $[g, L]=\{g(m)-m \mid m \in L\}$. It suffices to let $g$ run over a set of generators of the group $\mathcal{H}$ in the above formulas.

Lemma. With the above notation, $\mathbb{k}[L] / I_{\mathbb{k}[L]}(\mathcal{H}) \cong \mathbb{k}[L /[\mathcal{H}, L]]$ and

$$
\text { height } I_{\mathbb{k}[L]}(\mathcal{H})=\operatorname{rank}[\mathcal{H}, L]=\operatorname{rank} L-\operatorname{rank} L^{\mathcal{H}}
$$

Proof. Since the ideal $I_{\mathbb{k}[L]}(\mathcal{H})$ is generated by the elements $\mathbf{x}^{g(m)-m}-1$ with $m \in L$ and $g \in \mathcal{H}$, the isomorphism $\mathbb{k}[L] / I_{\mathbb{k}[L]}(\mathcal{H}) \cong \mathbb{k}[L /[\mathcal{H}, L]]$ is clear.

To prove the equality $\operatorname{rank}[\mathcal{H}, L]=\operatorname{rank} L-\operatorname{rank} L^{\mathcal{H}}$, note that the rational group algebra $\mathbb{Q}[\mathcal{H}]$ is the direct sum of the ideals $\mathbb{Q}\left(\sum_{g \in \mathcal{H}} g\right)$ and $\sum_{g \in \mathcal{H}} \mathbb{Q}(g-1)$. This implies $L \otimes_{\mathbb{Z}} \mathbb{Q}=\left(L^{\mathcal{H}} \otimes_{\mathbb{Z}} \mathbb{Q}\right) \oplus\left([\mathcal{H}, L] \otimes_{\mathbb{Z}} \mathbb{Q}\right)$. Hence, $\operatorname{rank} L=\operatorname{rank} L^{\mathcal{H}}+\operatorname{rank}[\mathcal{H}, L]$.

To complete the proof, it suffices to show that

$$
\text { height } \mathfrak{P}=\operatorname{rank}[\mathcal{H}, L]
$$

holds for any minimal covering prime $\mathfrak{P}$ of $I_{\mathbb{k}[L]}(\mathcal{H})$. Put $A=L /[\mathcal{H}, L]$ and $\overline{\mathfrak{P}}=$ $\mathfrak{P} / I_{\mathbb{k}[L]}(\mathcal{H})$, a minimal prime of $\mathbb{k}[L] / I_{\mathbb{k}[L]}(\mathcal{H})=\mathbb{k}[A]$. Further, put $\mathfrak{p}=\overline{\mathfrak{P}} \cap \mathbb{k}=$ $\mathfrak{P} \cap \mathbb{k}$. Since the extension $\mathbb{k} \hookrightarrow \mathbb{k}[A]=\mathbb{k}[L] / I_{\mathbb{k}[L]}(\mathcal{H})$ is free, $\mathfrak{p}$ is a minimal prime of $\mathbb{k}$; see $\mathrm{Bou}_{3}$, Cor. on p. AC VIII.15]. Hence, descending chains of primes in $\mathbb{k}[L]$ starting with $\mathfrak{P}$ correspond in a 1-to-1 fashion to descending chains of primes of $Q(\mathbb{k} / \mathfrak{p})[L]$ starting with the prime that is generated by $\mathfrak{P}$. Thus, replacing $\mathbb{k}$ by $Q(\mathbb{k} / \mathfrak{p})$, we may assume that $\mathbb{k}$ is a field. But then

$$
\text { height } \mathfrak{P}=\operatorname{dim} \mathbb{k}[L]-\operatorname{dim} \mathbb{k}[L] / \mathfrak{P}=\operatorname{rank} L-\operatorname{dim} \mathbb{k}[L] / \mathfrak{P} .
$$

Let $\overline{\mathfrak{P}}_{0}=\overline{\mathfrak{P}} \cap \mathbb{k}\left[A_{0}\right]$, where $A_{0}$ denotes the torsion subgroup of $A$. Since $\overline{\mathfrak{P}}$ is minimal, we have $\overline{\mathfrak{P}}=\overline{\mathfrak{P}}_{0} \mathbb{k}[A]$ and so $\mathbb{k}[L] / \mathfrak{P} \cong \mathbb{k}_{0}\left[A / A_{0}\right]$, where $\mathbb{k}_{0}=\mathbb{k}\left[A_{0}\right] / \overline{\mathfrak{P}}_{0}$ is a field. Thus, $\operatorname{dim} \mathbb{k}[L] / \mathfrak{P}=\operatorname{rank} A / A_{0}$. Finally, $\operatorname{rank} A / A_{0}=\operatorname{rank} A=\operatorname{rank} L-$ $\operatorname{rank}[\mathcal{H}, L]$, which completes the proof.

Specializing the lemma to the case where $\mathcal{H}=\langle g\rangle$ for some $g \in \mathcal{G}$, we see that $g$ acts as a $k$-reflection on $\mathbb{k}[L]$ if and only if $g$ acts as a $k$-reflection on $L$, that is,

$$
\operatorname{rank}[g, L] \leq k
$$


Moreover, the collection of subgroups $\mathfrak{X}_{k}$ in equation (1.1) can now be written as

$$
\mathfrak{X}_{k}=\left\{\mathcal{H} \leq \mathcal{G} \mid \operatorname{rank} L / L^{\mathcal{H}} \leq k\right\} \text {. }
$$

2.3. Fixed-point-free lattices for perfect groups. The $\mathcal{G}$-action on $L$ is called fixed-point-free if $g(m) \neq m$ holds for all $0 \neq m \in L$ and $1 \neq g \in \mathcal{G}$. Recall also that the group $\mathcal{G}$ is said to be perfect if $\mathcal{G}^{\text {ab }}=\mathcal{G} /[\mathcal{G}, \mathcal{G}]=1$.

Lemma. Assume that $\mathcal{G}$ is a non-trivial perfect group acting fixed-point-freely on the non-zero lattice $L$. Then $\mathcal{G}$ is isomorphic to the binary icosahedral group $2 . \mathcal{A}_{5} \cong$ $\mathrm{SL}_{2}\left(\mathbb{F}_{5}\right)$ and $\operatorname{rank} L$ is a multiple of 8.

Proof. Put $V=L \otimes_{\mathbb{Z}} \mathbb{C}$, a non-zero fixed-point-free $\mathbb{C}[\mathcal{G}]$-module. By a well-known theorem of Zassenhaus (see Wo, Theorem 6.2.1]), $\mathcal{G}$ is isomorphic to the binary icosahedral group 2. $\mathcal{A}_{5}$ and the irreducible constituents of $V$ are 2-dimensional. The binary icosahedral group has two irreducible complex representations of degree 2; they are Galois conjugates of each other and both have Frobenius-Schur indicator -1 . We denote the corresponding $\mathbb{C}[\mathcal{G}]$-modules by $V_{1}$ and $V_{2}$. Both $V_{i}$ occur with the same multiplicity in $V$, since $V$ is defined over $\mathbb{Q}$. Thus, $V \cong\left(V_{1} \oplus V_{2}\right)^{m}$ for some $m$ and $\operatorname{rank} L=4 m$. We have to show that $m$ is even. Since both $V_{i}$ have indicator -1 , it follows that $V_{1} \oplus V_{2}$ is not defined over $\mathbb{R}$, whereas each $V_{i}^{2}$ is defined over $\mathbb{R}$; see [I, (9.21)]. Thus, $V_{1} \oplus V_{2}$ represents an element $x$ of order 2 in the cokernel of the scalar extension map $G_{0}(\mathbb{R}[\mathcal{G}]) \rightarrow G_{0}(\mathbb{C}[\mathcal{G}])$, and $m x=0$. Therefore, $m$ must be even, as desired.

We remark that the binary icosahedral group $2 . \mathcal{A}_{5}$ is isomorphic to the subgroup of the non-zero quaternions $\mathbb{H}^{*}$ that is generated by $\left(a+i+j a^{*}\right) / 2$ and $\left(a+j+k a^{*}\right) / 2$, where $a=(1+\sqrt{5}) / 2$ and $a^{*}=(1-\sqrt{5}) / 2$ and $\{1, i, j, k\}$ is the standard $\mathbb{R}$ basis of $\mathbb{H}$. Thus, letting $2 . \mathcal{A}_{5}$ act on $\mathbb{H}$ via left multiplication, $\mathbb{H}$ becomes a 2-dimensional fixed-point-free complex representation of $2 . \mathcal{A}_{5}$. It is easy to see that this representation can be realized over $K=\mathbb{Q}(i, \sqrt{5})$; so $\mathbb{H}=V \otimes_{K} \mathbb{C}$ with $\operatorname{dim}_{\mathbb{Q}} V=2[K: \mathbb{Q}]=8$. Any $2 . \mathcal{A}_{5}$-lattice for $V$ will be fixed-point-free and have rank 8.

2.4. Isotropy groups. The isotropy group of an element $m \in L$ in $\mathcal{G}$ will be denoted by $\mathcal{G}_{m} ;$ so

$$
\mathcal{G}_{m}=\{g \in \mathcal{G} \mid g(m)=m\} .
$$

The $\mathcal{G}$-lattice $L$ is called faithful if $\operatorname{Ker}_{\mathcal{G}}(L)=\bigcap_{m \in L} \mathcal{G}_{m}=1$. The following lemma, at least part (a), is well known. We include the proof for the reader's convenience.

Lemma. (a) The set of isotropy groups $\left\{\mathcal{G}_{m} \mid m \in L\right\}$ is closed under conjugation and under taking intersections.

(b) Assume that the $\mathcal{G}$-lattice $L$ is faithful. If $\mathcal{G}_{m}(m \in L)$ is a minimal nonidentity isotropy group, then $\mathcal{G}_{m}$ acts fixed-point-freely on $L / L^{\mathcal{G}_{m}} \neq 0$.

Proof. Consider the $\mathbb{Q}[\mathcal{G}]$-module $V=L \otimes_{\mathbb{Z}} \mathbb{Q}$. The collection of isotropy groups $\mathcal{G}_{m}$ remains unchanged when allowing $m \in V$. Moreover, for any subgroup $\mathcal{H} \leq \mathcal{G}$, $L / L^{\mathcal{H}}$ is an $\mathcal{H}$-lattice with $L / L^{\mathcal{H}} \otimes_{\mathbb{Z}} \mathbb{Q} \cong V / V^{\mathcal{H}}$.

(a) The first assertion is clear, since ${ }^{g} \mathcal{G}_{m}=\mathcal{G}_{g(m)}$ holds for all $g \in \mathcal{G}, m \in V$. For the second assertion, let $M$ be a non-empty subset of $V$ and put $\mathcal{G}_{M}=\bigcap_{m \in M} \mathcal{G}_{m}$. We must show that $\mathcal{G}_{M}=\mathcal{G}_{m}$ for some $m \in V$. Put $W=V^{\mathcal{G}_{M}}$. If $g \in \mathcal{G} \backslash \mathcal{G}_{M}$, 
then $W^{g}=\{w \in W \mid g(w)=w\}$ is a proper subspace of $W$, since some element of $M$ does not belong to $W^{g}$. Any $m \in W \backslash \bigcup_{g \in \mathcal{G} \backslash \mathcal{G}_{M}} W^{g}$ satisfies $\mathcal{G}_{m}=\mathcal{G}_{M}$.

(b) Let $\mathcal{H}=\mathcal{G}_{m}$ be a minimal non-identity member of $\left\{\mathcal{G}_{m} \mid m \in V\right\}$. As $\mathbb{Q}[\mathcal{H}]$-modules, we may identify $V$ and $V^{\mathcal{H}} \oplus V / V^{\mathcal{H}}$. If $0 \neq v \in V / V^{\mathcal{H}}$, then $\mathcal{H}_{v}=\mathcal{H} \cap \mathcal{G}_{v} \subsetneq \mathcal{H}$. In view of (a), our minimality assumption on $\mathcal{H}$ forces $\mathcal{H}_{v}=1$. Thus, $\mathcal{H}$ acts fixed-point-freely on $V / V^{\mathcal{H}}$, and hence on $L / L^{\mathcal{H}}$.

Proposition. Assume that $L$ is a faithful $\mathcal{G}$-lattice such that all minimal isotropy groups $1 \neq \mathcal{G}_{m}(m \in L)$ are perfect. Then $\operatorname{rank} L / L^{\mathcal{H}} \geq 8$ holds for every nonidentity subgroup $\mathcal{H} \leq \mathcal{G}$.

In the notation of equation (2.2), the conclusion of the proposition can be stated as follows:

$$
\mathfrak{X}_{k}=\{1\} \text { for all } k<8 \text {. }
$$

Proof of the Proposition. Put $\overline{\mathcal{H}}=\bigcap_{m \in L^{\mathcal{H}}} \mathcal{G}_{m}$. Then $\bar{H} \supseteq \mathcal{H}$ and $L^{\overline{\mathcal{H}}}=L^{\mathcal{H}}$. Lemma 2.4 (a) further implies that $\overline{\mathcal{H}}=\mathcal{G}_{m}$ for some $m$. Replacing $\mathcal{H}$ by $\bar{H}$, we may assume that $\mathcal{H}$ is a nonidentity isotropy group. If $\mathcal{H}$ is not minimal then replace $\mathcal{H}$ by a smaller nonidentity isotropy group; this does not increase the value of $\operatorname{rank} L / L^{\mathcal{H}}$. Thus, we may assume that $\mathcal{H}$ is a minimal nonidentity isotropy group, and hence $\mathcal{H}$ is perfect. By Lemma $2.4(\mathrm{~b}), \mathcal{H}$ acts fixed-point-freely on $L / L^{\mathcal{H}} \neq 0$ and Lemma 2.3 implies that $\operatorname{rank} L / L^{\mathcal{H}} \geq 8$, proving the proposition.

2.5. Cohomology. Let $\mathfrak{X}$ denote any collection of subgroups of $\mathcal{G}$ that is closed under conjugation and under taking subgroups. We will investigate injectivity of the restriction map

$$
\operatorname{res}_{\mathfrak{X}}^{\mathcal{G}}: H^{k}(\mathcal{G}, \mathbb{k}[L]) \rightarrow \prod_{\mathcal{H} \in \mathfrak{X}} H^{k}(\mathcal{H}, \mathbb{k}[L]) .
$$

This map was considered in Proposition 1.4 for $\mathfrak{X}=\mathfrak{X}_{k+1}$.

Lemma. The map $\operatorname{res}_{\mathfrak{X}}^{\mathcal{G}}: H^{k}(\mathcal{G}, \mathbb{k}[L]) \rightarrow \prod_{\mathcal{H} \in \mathfrak{X}} H^{k}(\mathcal{H}, \mathbb{k}[L])$ is injective if and only if the restriction maps

$$
H^{k}\left(\mathcal{G}_{m}, \mathbb{k}\right) \rightarrow \prod_{\substack{\mathcal{H} \in \mathcal{X} \\ \mathcal{H} \leq \mathcal{G}_{m}}} H^{k}(\mathcal{H}, \mathbb{k})
$$

are injective for all $m \in L$.

Proof. As $\mathbb{k}[\mathcal{G}]$-module, $\mathbb{k}[L]$ is a permutation module:

$$
\mathbb{k}[L] \cong \bigoplus_{m \in \mathcal{G} \backslash L} \mathbb{k}\left[\mathcal{G} / \mathcal{G}_{m}\right]
$$

where $\mathbb{k}\left[\mathcal{G} / \mathcal{G}_{m}\right]=\mathbb{k}[\mathcal{G}] \otimes_{\mathbb{k}\left[\mathcal{G}_{m}\right]} \mathbb{k}$ and $\mathcal{G} \backslash L$ is a transversal for the $\mathcal{G}$-orbits in $L$. For each subgroup $\mathcal{H} \leq \mathcal{G}$,

$$
\left.\mathbb{k}\left[\mathcal{G} / \mathcal{G}_{m}\right]\right|_{\mathcal{H}} \cong \bigoplus_{g \in \mathcal{H} \backslash \mathcal{G} / \mathcal{G}_{m}} \mathbb{k}\left[\mathcal{H} /{ }^{g} \mathcal{G}_{m} \cap \mathcal{H}\right]
$$

see $[\mathrm{CR}, 10.13]$. Therefore, $\operatorname{res}_{\mathcal{H}}^{\mathcal{G}}$ is the direct sum of the restriction maps

$$
H^{k}\left(\mathcal{G}, \mathbb{k}\left[\mathcal{G} / \mathcal{G}_{m}\right]\right) \rightarrow H^{k}\left(\mathcal{H}, \mathbb{k}\left[\mathcal{G} / \mathcal{G}_{m}\right]\right)=\bigoplus_{g \in \mathcal{H} \backslash \mathcal{G} / \mathcal{G}_{m}} H^{k}\left(\mathcal{H}, \mathbb{k}\left[\mathcal{H} /{ }^{g} \mathcal{G}_{m} \cap \mathcal{H}\right]\right)
$$


By the Eckmann-Shapiro Lemma $[\mathrm{Br}, \operatorname{III}(5.2),(6.2)], H^{k}\left(\mathcal{G}, \mathbb{k}\left[\mathcal{G} / \mathcal{G}_{m}\right]\right) \cong H^{k}\left(\mathcal{G}_{m}, \mathbb{k}\right)$ and $H^{k}\left(\mathcal{H}, \mathbb{k}\left[\mathcal{H} /{ }^{g} \mathcal{G}_{m} \cap \mathcal{H}\right]\right) \cong H^{k}\left({ }^{g} \mathcal{G}_{m} \cap \mathcal{H}, \mathbb{k}\right)$. In terms of these isomorphisms, the above restriction map becomes

$$
\begin{aligned}
\rho_{\mathcal{H}, m}: H^{k}\left(\mathcal{G}_{m}, \mathbb{k}\right) & \rightarrow & \bigoplus_{g \in \mathcal{H} \backslash \mathcal{G} / \mathcal{G}_{m}} H^{k}\left({ }^{g} \mathcal{G}_{m} \cap \mathcal{H}, \mathbb{k}\right) \\
{[f] } & \mapsto & \left(\left[\underline{h} \mapsto f\left(g^{-1} \underline{h} g\right)\right]\right)_{g}
\end{aligned}
$$

where [.] denotes the cohomology class of a $k$-cocycle and $\underline{h}$ stands for a $k$-tuple of elements of ${ }^{g} \mathcal{G}_{m} \cap \mathcal{H}$. Therefore,

$$
\operatorname{Ker} \rho_{\mathcal{H}, m}=\bigcap_{g \in \mathcal{H} \backslash \mathcal{G} / \mathcal{G}_{m}} \operatorname{Ker}\left(\operatorname{res}_{\mathcal{G}_{m} \cap \mathcal{H}^{g}}^{\mathcal{G}_{m}}: H^{k}\left(\mathcal{G}_{m}, \mathbb{k}\right) \rightarrow H^{k}\left(\mathcal{G}_{m} \cap \mathcal{H}^{g}, \mathbb{k}\right)\right)
$$

Thus, Ker res ${ }_{\mathfrak{X}}^{\mathcal{G}}$ is isomorphic to the direct sum of the kernels of the restriction maps

$$
H^{k}\left(\mathcal{G}_{m}, \mathbb{k}\right) \rightarrow \prod_{\mathcal{H} \in \mathfrak{X}} H^{k}\left(\mathcal{G}_{m} \cap \mathcal{H}^{g}, \mathbb{k}\right)
$$

with $m \in \mathcal{G} \backslash L$. Finally, by hypothesis on $\mathfrak{X}$, the groups $\mathcal{G}_{m} \cap \mathcal{H}^{g}$ with $\mathcal{H} \in \mathfrak{X}$ are exactly the groups $\mathcal{H} \in \mathfrak{X}$ with $\mathcal{H} \leq \mathcal{G}_{m}$. The lemma follows.

Corollary. Let $\mathbb{k}=\mathbb{Z} /(|\mathcal{G}|)$ and $k=1$. Then $\operatorname{res}_{\mathfrak{X}}^{\mathcal{G}}$ injective if and only if all $\mathcal{G}_{m}^{\mathrm{ab}}$ $(m \in L)$ are generated by the images of the subgroups $\mathcal{H} \leq \mathcal{G}_{m}$ with $\mathcal{H} \in \mathfrak{X}$.

Proof. By the lemma with $k=1$, the hypothesis on the restriction map says that all restrictions

$$
H^{1}\left(\mathcal{G}_{m}, \mathbb{k}\right) \rightarrow \prod_{\substack{\mathcal{H} \in \mathcal{X} \\ \mathcal{H} \leq \mathcal{G}_{m}}} H^{1}(\mathcal{H}, \mathbb{k})
$$

are injective. Now, for each $\mathcal{H} \leq \mathcal{G}, H^{1}(\mathcal{H}, \mathbb{k})=\operatorname{Hom}\left(\mathcal{H}^{\mathrm{ab}}, \mathbb{k}\right) \cong \mathcal{H}^{\text {ab }}$, where the last isomorphism holds by our choice of $\mathbb{k}$. Therefore, injectivity of the above map is equivalent to $\mathcal{G}_{m}^{\mathrm{ab}}$ being generated by the images of all $\mathcal{H} \leq \mathcal{G}_{m}$ with $\mathcal{H} \in \mathfrak{X}$.

\section{The Cohen-Macaulay property}

3.1. Continuing with the notation of $\$ 2.1$ we now turn to the question of when the invariant algebra $\mathbb{k}[L]^{\mathcal{G}}$ is Cohen-Macaulay. Our principal tool will be Proposition 1.4. We remark that the Cohen-Macaulay hypothesis of Proposition 1.4 simplifies slightly in the setting of multiplicative actions: it suffices to assume that $\mathbb{k}[L]^{\mathcal{G}}$ is Cohen-Macaulay. Indeed, in this case the base ring $\mathbb{k}$ is also Cohen-Macaulay, because $\mathbb{k}[L]^{\mathcal{G}}$ is free over $\mathbb{k}$, and then $\mathbb{k}[L]$ is Cohen-Macaulay as well; see BH, Exercise 2.1.23 and Theorems 2.1.9, 2.1.3(b)].

3.2. Base rings. Our main interest is in the case where $\mathbb{k}=\mathbb{Z}$. As the following lemma shows, if $\mathbb{Z}[L]^{\mathcal{G}}$ is Cohen-Macaulay, then so is $\mathbb{k}[L]^{\mathcal{G}}$ for any Cohen-Macaulay base ring $\mathbb{k}$.

Lemma. The following are equivalent:

(a) $\mathbb{Z}[L]^{\mathcal{G}}$ is Cohen-Macaulay;

(b) $\mathbb{k}[L]^{\mathcal{G}}$ is Cohen-Macaulay whenever $\mathbb{k}$ is;

(c) $\mathbb{k}[L]^{\mathcal{G}}$ is Cohen-Macaulay for $\mathbb{k}=\mathbb{Z} /(|\mathcal{G}|)$;

(d) $\mathbb{F}_{p}[L]^{\mathcal{G}}$ is Cohen-Macaulay for all primes $p$ dividing $|\mathcal{G}|$. 
Proof. (a) $\Rightarrow$ (b): Put $S=\mathbb{k}[L]^{\mathcal{G}}$ and consider the extension of rings $\mathbb{k} \hookrightarrow S$. This extension is free; see \$2.1. By [BH, Exercise 2.1.23], $S$ is Cohen-Macaulay if (and only if) $\mathbb{k}$ is Cohen-Macaulay and, for all $\mathfrak{P} \in \operatorname{Spec} S$, the fibre $S_{\mathfrak{P}} / \mathfrak{p} S_{\mathfrak{P}}$ is Cohen-Macaulay, where $\mathfrak{p}=\mathfrak{P} \cap \mathbb{k}$. But $S_{\mathfrak{P}} / \mathfrak{p} S_{\mathfrak{P}}$ is a localization of $(S / \mathfrak{p} S)_{\mathfrak{p} \backslash 0} \cong$ $Q(\mathbb{k} / \mathfrak{p})[L]^{\mathcal{G}} ;$ see equation (2.1). Therefore, by $[\mathrm{BH}$, Theorem 2.1.3(b)], it suffices to show that $Q(\mathbb{k} / \mathfrak{p})[L]^{\mathcal{G}}$ is Cohen-Macaulay. In other words, we may assume that $\mathbb{k}$ is a field. By [BH], Theorem 2.1.10], we may further assume that $\mathbb{k}=\mathbb{Q}$ or $\mathbb{k}=\mathbb{F}_{p}$. But equation (2.1) implies that $\mathbb{Q}[L]^{\mathcal{G}}=\mathbb{Z}[L]_{\mathbb{Z} \backslash 0}^{\mathcal{G}}$ and $\mathbb{F}_{p}[L]^{\mathcal{G}} \cong \mathbb{Z}[L]^{\mathcal{G}} /(p)$. Since $\mathbb{Z}[L]^{\mathcal{G}}$ is assumed Cohen-Macaulay, $\left[\mathrm{BH}\right.$, Theorem 2.1.3] implies that $\mathbb{Q}[L]^{\mathcal{G}}$ and $\mathbb{F}_{p}[L]^{\mathcal{G}}$ are Cohen-Macaulay, as desired.

(b) $\Rightarrow$ (c) is clear.

(c) $\Rightarrow(\mathrm{d})$ : Write $|\mathcal{G}|=\prod_{p} p^{n_{p}}$. Then $\mathbb{k}[L] \cong \prod_{p} \mathbb{Z} /\left(p^{n_{p}}\right)[L]^{\mathcal{G}}$ and $\mathbb{Z} /\left(p^{n_{p}}\right)[L]^{\mathcal{G}}$ is a localization of $\mathbb{k}[L]^{\mathcal{G}}$. Therefore, $\mathbb{Z} /\left(p^{n_{p}}\right)[L]^{\mathcal{G}}$ is Cohen-Macaulay, by $[\mathrm{BH}$, Theorem 2.1.3(b)]. If $n_{p} \neq 0$, then it follows from [BH, Theorem 2.1.3(a)] that $\mathbb{Z}_{(p)}[L]^{\mathcal{G}}$ and $\mathbb{F}_{p}[L]^{\mathcal{G}} \cong \mathbb{Z}_{(p)}[L]^{\mathcal{G}} /(p)$ are Cohen-Macaulay.

(d) $\Rightarrow\left(\right.$ a): First, (d) implies that $\mathbb{F}_{p}[L]^{\mathcal{G}}$ is Cohen-Macaulay for all primes $p$. For, if $p$ does not divide $|\mathcal{G}|$, then $\mathbb{F}_{p}[L]^{\mathcal{G}}$ is always Cohen-Macaulay; see [BH], Corollary 6.4.6]. Now let $\mathfrak{P}$ be a maximal ideal of $\mathbb{Z}[L]$. Then $\mathfrak{P} \cap \mathbb{Z}=(p)$ for some prime $p$ and $\mathbb{Z}[L]_{\mathfrak{P}}^{\mathcal{G}} /(p)$ is a localization of $\mathbb{Z}[L]^{\mathcal{G}} /(p)=\mathbb{F}_{p}[L]^{\mathcal{G}}$. Thus, $\mathbb{Z}[L]_{\mathfrak{P}}^{\mathcal{G}} /(p)$ is Cohen-Macaulay and $\left[\mathrm{BH}\right.$, Theorem 2.1.3(a)] further implies that $\mathbb{Z}[L]_{\mathfrak{P}}^{\mathcal{G}}$ is Cohen-Macaulay. Since, $\mathfrak{P}$ was arbitrary, (a) is proved.

Since normal rings of (Krull) dimension at most 2 are Cohen-Macaulay, the implication $(\mathrm{d}) \Rightarrow(\mathrm{b})$ of the lemma shows that $\mathbb{k}[L]^{\mathcal{G}}$ is certainly Cohen-Macaulay whenever $\mathbb{k}$ is Cohen-Macaulay and $L$ has rank at most 2 .

3.3. Proof of the Theorem. We are now ready to prove the Theorem stated in the Introduction. Recall that, for any subgroup $\mathcal{H} \leq \mathcal{G}, \mathcal{H}^{(2)}$ denotes the subgroup generated by the elements of $\mathcal{H}$ that act as bireflections on $L$ or, equivalently, by the subgroups of $\mathcal{H}$ that belong to $\mathfrak{X}_{2}$; see (2.2). Throughout, we assume that $\mathbb{Z}[L]^{\mathcal{G}}$ is Cohen-Macaulay.

We first show that $\mathcal{G}_{m} / \mathcal{G}_{m}^{(2)}$ is a perfect group for all $m \in L$. Put $\mathbb{k}=\mathbb{Z} /(|\mathcal{G}|)$. Then $\mathbb{k}[L]^{\mathcal{G}}$ is Cohen-Macaulay, by Lemma 3.2. Therefore, the restriction

$$
H^{1}(\mathcal{G}, \mathbb{k}[L]) \rightarrow \prod_{\mathcal{H} \in \mathfrak{X}_{2}} H^{1}(\mathcal{H}, \mathbb{k}[L])
$$

is injective, by Proposition 1.4 see the remark in $\$ 3.1$. Corollary 2.5 yields that all $\mathcal{G}_{m}^{\text {ab }}$ are generated by the images of the subgroups $\mathcal{H} \leq \mathcal{G}_{m}$ with $\mathcal{H} \in \mathfrak{X}_{2}$. In other words, each $\mathcal{G}_{m}^{\text {ab }}$ is generated by the images of the bireflections in $\mathcal{G}_{m}$. Therefore, $\left(\mathcal{G}_{m} / \mathcal{G}_{m}^{(2)}\right)^{\mathrm{ab}}=1$, as desired.

Now assume that $\mathcal{G}$ acts non-trivially on $L$. Our goal is to show that some isotropy group $\mathcal{G}_{m}$ is non-perfect. Suppose otherwise. Replacing $\mathcal{G}$ by $\mathcal{G} / \operatorname{Ker}_{\mathcal{G}}(L)$ we may assume that $1 \neq \mathcal{G}$ acts faithfully on $L$. Then $\mathfrak{X}_{k}=\{1\}$ for all $k<8$, by Proposition 2.4. It follows that

$$
k=\inf \left\{i>0 \mid H^{i}(\mathcal{G}, \mathbb{k}[L]) \neq 0\right\} \geq 7 .
$$

Indeed, if $k<7$, then Proposition 1.4 implies that $0 \neq H^{k}(\mathcal{G}, \mathbb{k}[L])$ embeds into $\prod_{\mathcal{H} \in \mathfrak{X}_{k+1}} H^{k}(\mathcal{H}, \mathbb{k}[L])$ which is trivial, because $\mathfrak{X}_{k+1}=\{1\}$. By Lemma 2.5 with 
$\mathfrak{X}=\{1\}$, we conclude that

$$
H^{i}\left(\mathcal{G}_{m}, \mathbb{k}\right)=0 \text { for all } m \in L \text { and all } 0<i<7 .
$$

On the other hand, choosing $\mathcal{G}_{m}$ minimal with $\mathcal{G}_{m} \neq 1$, we know by Lemmas 2.3 and 2.4 (b) that $\mathcal{G}_{m}$ is isomorphic to the binary icosahedral group 2. $\mathcal{A}_{5}$. The cohomology of $2 . \mathcal{A}_{5}$ is 4-periodic (see $\left[\mathrm{Br}\right.$, p. 155]). Hence, $H^{3}\left(\mathcal{G}_{m}, \mathbb{k}\right) \cong H^{-1}\left(\mathcal{G}_{m}, \mathbb{k}\right)=$ $\operatorname{ann}_{\mathbb{k}}\left(\sum_{\mathcal{G}_{m}} g\right) \cong \mathbb{Z} /\left(\left|\mathcal{G}_{m}\right|\right) \neq 0$. This contradiction completes the proof of the Theorem.

3.4. Rational invariance. We now show that the Cohen-Macaulay property of $\mathbb{k}[L]^{\mathcal{G}}$ depends only on the rational isomorphism class of the $\mathcal{G}$-lattice $L$. Recall that $\mathcal{G}$-lattices $L$ and $L^{\prime}$ are said to be rationally isomorphic if $L \otimes_{\mathbb{Z}} \mathbb{Q} \cong L^{\prime} \otimes_{\mathbb{Z}} \mathbb{Q}$ as $\mathbb{Q}[\mathcal{G}]$-modules. In this section, $\mathbb{k}$ denotes any commutative base ring.

Proposition. If $\mathbb{k}[L]^{\mathcal{G}}$ is Cohen-Macaulay, then so is $\mathbb{k}\left[L^{\prime}\right]^{\mathcal{G}}$ for any $\mathcal{G}$-lattice $L^{\prime}$ that is rationally isomorphic to $L$.

Proof. Assume that $L \otimes_{\mathbb{Z}} \mathbb{Q} \cong L^{\prime} \otimes_{\mathbb{Z}} \mathbb{Q}$. Replacing $L^{\prime}$ by an isomorphic copy inside $L \otimes_{\mathbb{Z}} \mathbb{Q}$, we may assume that $L \supseteq L^{\prime}$ and $L / L^{\prime}$ is finite. Then $\mathbb{k}[L]$ is finite over $\mathbb{k}\left[L^{\prime}\right]$ which in turn is integral over $\mathbb{k}\left[L^{\prime}\right]^{\mathcal{G}}$. Therefore, $\mathbb{k}[L]$ is integral over $\mathbb{k}\left[L^{\prime}\right]^{\mathcal{G}}$, and hence so is $\mathbb{k}[L]^{\mathcal{G}}$.

We now invoke a ring-theoretic result of Hochster and Eagon $\mathrm{HE}$ (or see $\mathrm{BH}$, Theorem 6.4.5]): Let $R \supseteq S$ be an integral extension of commutative rings having a Reynolds operator, that is, an $S$-linear map $R \rightarrow S$ that restricts to the identity on $S$. If $R$ is Cohen-Macaulay, then $S$ is Cohen-Macaulay as well.

To construct the requisite Reynolds operator, consider the truncation map

$$
\pi: \mathbb{k}[L] \rightarrow \mathbb{k}\left[L^{\prime}\right], \quad \sum_{m \in L} k_{m} \mathbf{x}^{m} \mapsto \sum_{m \in L^{\prime}} k_{m} \mathbf{x}^{m} .
$$

This is a Reynolds operator for the extension $\mathbb{k}[L] \supseteq \mathbb{k}\left[L^{\prime}\right]$ that satisfies $\pi(g(f))=$ $g(\pi(f))$ for all $g \in \mathcal{G}, f \in \mathbb{k}[L]$. Therefore, $\pi$ restricts to a Reynolds operator $\mathbb{k}[L]^{\mathcal{G}} \rightarrow \mathbb{k}\left[L^{\prime}\right]^{\mathcal{G}}$ and the proposition follows.

The proposition in particular allows us to reduce the general case of the CohenMacaulay problem for multiplicative invariants to the case of effective $\mathcal{G}$-lattices. Recall that the $\mathcal{G}$-lattice $L$ is effective if $L^{\mathcal{G}}=0$. For any $\mathcal{G}$-lattice $L$, the quotient $L / L^{\mathcal{G}}$ is an effective $\mathcal{G}$-lattice; this follows, for example, from the fact that $L$ is rationally isomorphic to the $\mathcal{G}$-lattice $L^{\mathcal{G}} \oplus L / L^{\mathcal{G}}$.

Corollary. $\mathbb{k}[L]^{\mathcal{G}}$ is Cohen-Macaulay if and only if this holds for $\mathbb{k}\left[L / L^{\mathcal{G}}\right]^{\mathcal{G}}$.

Proof. By the proposition, we may replace $L$ by $L^{\prime}=L^{\mathcal{G}} \oplus L / L^{\mathcal{G}}$. But $\mathbb{k}\left[L^{\prime}\right]^{G} \cong$ $\mathbb{k}\left[L / L^{\mathcal{G}}\right]^{\mathcal{G}} \otimes_{\mathbb{k}} \mathbb{k}\left[L^{\mathcal{G}}\right]$, a Laurent polynomial algebra over $k\left[L / L^{\mathcal{G}}\right]^{\mathcal{G}}$. Thus, by $[\mathrm{BH}$, Theorems 2.1.3 and 2.1.9], $\mathbb{k}\left[L^{\prime}\right]^{\mathcal{G}}$ is Cohen-Macaulay if and only if $\mathbb{k}\left[L / L^{\mathcal{G}}\right]^{\mathcal{G}}$ is Cohen-Macaulay. The corollary follows.

\subsection{Remarks and examples.}

3.5.1. Abelian bireflection groups. It is not hard to show that if $\mathcal{G}$ is a finite abelian group acting as a bireflection group on the lattice $L$, then $\mathbb{Z}[L]^{\mathcal{G}}$ is Cohen-Macaulay. Using Corollary 3.4 and an induction on $\operatorname{rank} L$, the proof reduces to the verification 
that $\mathbb{Z}[L]^{\mathcal{G}}$ is Cohen-Macaulay for $L=\mathbb{Z}^{n}$ and $\mathcal{G}=\operatorname{diag}( \pm 1, \ldots, \pm 1) \cap \mathrm{SL}_{n}(\mathbb{Z})$. Direct computation shows that, for $n \geq 2$,

$$
\mathbb{Z}[L]^{\mathcal{G}}=\mathbb{Z}\left[\xi_{1}, \ldots, \xi_{n}\right] \oplus \eta \mathbb{Z}\left[\xi_{1}, \ldots, \xi_{n}\right],
$$

where $\xi_{i}=\mathbf{x}^{e_{i}}+\mathbf{x}^{-e_{i}}$ is the $\mathcal{G}$-orbit sum of the standard basis element $e_{i} \in \mathbb{Z}^{n}$ and $\eta$ is the orbit sum of $\sum_{i} e_{i}=(1, \ldots, 1)$.

It would be worthwhile to try and extend this result to larger classes of bireflection groups. The aforementioned classification of bireflection groups in GuS will presumably be helpful in this endeavor.

3.5.2. Subgroups of reflection groups. Assume that $\mathcal{G}$ acts as a reflection group on the lattice $L$ and let $\mathcal{H}$ be a subgroup of $\mathcal{G}$ with $[\mathcal{G}: \mathcal{H}]=2$. Then $\mathcal{H}$ acts as a bireflection group. (More generally, if $\mathcal{G}$ acts as a $k$-reflection group and $[\mathcal{G}: \mathcal{H}]=m$, then $\mathcal{H}$ acts as a $k m$-reflection group; see $\left[\mathrm{L}_{1}\right.$.) Presumably $\mathbb{Z}[L]^{\mathcal{H}}$ will always be Cohen-Macaulay, but I have no proof. For an explicit example, let $\mathcal{G}=\mathcal{S}_{n}$ be the symmetric group on $\{1, \ldots, n\}$ and let $L=U_{n}$ be the standard permutation lattice for $\mathcal{S}_{n}$; so $U_{n}=\bigoplus_{i=1}^{n} \mathbb{Z} e_{i}$ with $s\left(e_{i}\right)=e_{s(i)}$ for $s \in \mathcal{S}_{n}$. Transpositions act as reflections on $U_{n}$ and 3 -cycles as bireflections. Let $\mathcal{A}_{n} \leq \mathcal{S}_{n}$ denote the alternating group. To compute $\mathbb{Z}\left[U_{n}\right]^{\mathcal{A}_{n}}$, put $x_{i}=\mathbf{x}^{e_{i}} \in \mathbb{Z}\left[U_{n}\right]$. Then $\mathbb{Z}\left[U_{n}\right]=\mathbb{Z}\left[x_{1}, \ldots, x_{n}\right]\left[s_{n}^{-1}\right]$, where $s_{n}=\mathbf{x}^{\sum_{1}^{n} e_{i}}=\prod_{1}^{n} x_{i}$ is the $n^{\text {th }}$ elementary symmetric function, and $\mathcal{S}_{n}$ acts via $s\left(x_{i}\right)=x_{s(i)}\left(s \in \mathcal{S}_{n}\right)$. Therefore, $\mathbb{Z}\left[U_{n}\right]^{\mathcal{A}_{n}}=\mathbb{Z}\left[x_{1}, \ldots, x_{n}\right]^{\mathcal{A}_{n}}\left[s_{n}^{-1}\right]$. The ring $\mathbb{Z}\left[x_{1}, \ldots, x_{n}\right]^{\mathcal{A}_{n}}$ of polynomial $\mathcal{A}_{n}$-invariants has the following form; see $\underline{\underline{S}}$, Theorem 1.3.5]: $\mathbb{Z}\left[x_{1}, \ldots, x_{n}\right]^{\mathcal{A}_{n}}=\mathbb{Z}\left[s_{1}, \ldots, s_{n}\right] \oplus d \mathbb{Z}\left[s_{1}, \ldots, s_{n}\right]$, where $s_{i}$ is the $i^{\text {th }}$ elementary symmetric function and

$$
d=\frac{1}{2}\left(\Delta+\Delta_{+}\right)
$$

with $\Delta_{+}=\prod_{i<j}\left(x_{i}+x_{j}\right)$ and $\Delta=\prod_{i<j}\left(x_{i}-x_{j}\right)$, the Vandermonde determinant. Thus,

$$
\mathbb{Z}\left[U_{n}\right]^{\mathcal{A}_{n}}=\mathbb{Z}\left[s_{1}, \ldots, s_{n-1}, s_{n}^{ \pm 1}\right] \oplus d \mathbb{Z}\left[s_{1}, \ldots, s_{n-1}, s_{n}^{ \pm 1}\right] .
$$

This is Cohen-Macaulay, being free over $\mathbb{Z}\left[s_{1}, \ldots, s_{n-1}, s_{n}^{ \pm 1}\right]$.

3.5.3. $\mathcal{S}_{n}$-lattices. If $L$ is a lattice for the symmetric group $\mathcal{S}_{n}$ such that $\mathbb{Z}[L]^{\mathcal{S}_{n}}$ is Cohen-Macaulay, then the Theorem implies that $\mathcal{S}_{n}$ acts as a bireflection group on $L$, and hence on all simple constituents of $L \otimes_{\mathbb{Z}} \mathbb{Q}$. The simple $\mathbb{Q}\left[\mathcal{S}_{n}\right]$-modules are the Specht modules $S^{\lambda}$ for partitions $\lambda$ of $n$. If $n \geq 7$, then the only partitions $\lambda$ so that $\mathcal{S}_{n}$ acts as a bireflection group on $S^{\lambda}$ are $(n),\left(1^{n}\right)$ and $(n-1,1)$; this follows from the lists in $[\mathrm{Hu}$ and $[\mathrm{W}$. The corresponding Specht modules are trivial module, $\mathbb{Q}$, the sign module $\mathbb{Q}^{-}$, and the rational root module $A_{n-1} \otimes_{\mathbb{Z}} \mathbb{Q}$, where $A_{n-1}=\left\{\sum_{i} z_{i} e_{i} \in U_{n} \mid \sum_{i} z_{i}=0\right\}$ and $U_{n}$ is as in 33.5 .2 . Thus, if $n \geq 7$ and $\mathbb{Z}[L]^{\mathcal{S}_{n}}$ is Cohen-Macaulay, then we must have

$$
L \otimes_{\mathbb{Z}} \mathbb{Q} \cong \mathbb{Q}^{r} \oplus\left(\mathbb{Q}^{-}\right)^{s} \oplus\left(A_{n-1} \otimes_{\mathbb{Z}} \mathbb{Q}\right)^{t}
$$

with $s+t \leq 2$. In most cases, $\mathbb{Z}[L]^{\mathcal{S}_{n}}$ is easily seen to be Cohen-Macaulay. Indeed, we may assume $r=0$ by Corollary 3.4. If $s+t \leq 1$, then $\mathcal{S}_{n}$ acts as a reflection group on $L$ and so $\mathbb{Z}[L]^{\mathcal{S}_{n}}$ is Cohen-Macaulay by $\left[\mathrm{L}_{2}\right.$. For $t=0$ we may quote the last remark in 33.2 . This leaves the cases $s=t=1$ and $s=0, t=2$ to consider. 
If $s=t=1$, then add a copy of $\mathbb{Q}$ so that $L$ is rationally isomorphic to $U_{n} \oplus \mathbb{Z}^{-}$. Using the notation of $\$ 3.5 .2$ and putting $t=\mathbf{x}^{\left(0_{U_{n}}, 1\right)} \in \mathbb{Z}\left[U_{n} \oplus \mathbb{Z}^{-}\right]$the invariants are

$$
\mathbb{Z}\left[U_{n} \oplus \mathbb{Z}^{-}\right]^{\mathcal{S}_{n}}=R \oplus R \varphi
$$

with $R=\mathbb{Z}\left[s_{1}, \ldots, s_{n-1}, s_{n}^{ \pm 1}, t+t^{-1}\right]$ and $\varphi=\frac{1}{2}\left(\Delta_{+}+\Delta\right) t+\frac{1}{2}\left(\Delta_{+}-\Delta\right) t^{-1}$.

If $s=0$ and $t=2$, then we may replace $L$ by the lattice $U_{n}^{2}=U_{n} \oplus U_{n}$. By Lemma $\left[3.2 \mathbb{Z}\left[U_{n}^{2}\right]^{\mathcal{S}_{n}}\right.$ is Cohen-Macaulay precisely if $\mathbb{F}_{p}\left[U_{n}^{2}\right]^{\mathcal{S}_{n}}$ is Cohen-Macaulay for all primes $p \leq n$. As in $\$ 3.5 .2$, one sees that $\mathbb{F}_{p}\left[U_{n}^{2}\right]^{\mathcal{S}_{n}}$ is a localization of the algebra "vector invariants" $\mathbb{F}_{p}\left[x_{1}, \ldots, x_{n}, y_{1}, \ldots, y_{n}\right]^{\mathcal{S}_{n}}$. By $\left[\mathrm{K}_{2}\right.$, Corollary 3.5], this algebra is known to be Cohen-Macaulay for $n / 2<p \leq n$, but the primes $p \leq n / 2$ apparently remain to be dealt with.

3.5.4. Ranks $\leq 4$. As was pointed out in $\$ 3.2, \mathbb{Z}[L]^{\mathcal{G}}$ is always Cohen-Macaulay when $\operatorname{rank} L \leq 2$.

For $L=\mathbb{Z}^{3}$, there are $32 \mathbb{Q}$-classes of finite subgroups $\mathcal{G} \leq \mathrm{GL}_{3}(\mathbb{Z})$. All $\mathcal{G}$ are solvable; in fact, their orders divide 48. The Sylow 3 -subgroup $\mathcal{H} \leq \mathcal{G}$, if nontrivial, is generated by a bireflection of order 3. Thus, $\mathbb{F}_{3}[L]^{\mathcal{H}}$ is Cohen-Macaulay, and hence so is $\mathbb{F}_{3}[L]^{\mathcal{G}}$. Therefore, by Lemma $\left[3.2, \mathbb{Z}[L]^{\mathcal{G}}\right.$ is Cohen-Macaulay if and only if $\mathbb{F}_{2}[L]^{\mathcal{G}}$ is Cohen-Macaulay, and for this to occur, $\mathcal{G}$ must be generated by bireflections. It turns out that 3 of the $32 \mathbb{Q}$-classes consist of non-bireflection groups; these classes are represented by the cyclic groups

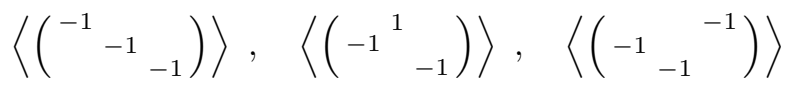

of orders 2, 4 and 6 (the latter two classes each split into $2 \mathbb{Z}$-classes). For the $\mathbb{Q}$-classes consisting of bireflection groups, Pathak $[\mathrm{Pk}]$ has checked explicitly that $\mathbb{F}_{2}[L]^{\mathcal{G}}$ is indeed Cohen-Macaulay.

In rank 4 , there are $227 \mathbb{Q}$-classes of finite subgroups $\mathcal{G} \leq \mathrm{GL}_{4}(\mathbb{Z})$. All but 5 of them consist of solvable groups and 4 of the non-solvable classes are bireflection groups, the one exception being represented by $\mathcal{S}_{5}$ acting on the signed root lattice $\mathbb{Z}^{-} \otimes_{\mathbb{Z}} A_{4}$. Thus, if the group $\mathcal{G} / \mathcal{G}^{(2)}$ is perfect, then it is actually trivial, that is, $\mathcal{G}$ is a bireflection group. It also turns out that, in this case, all isotropy groups $\mathcal{G}_{m}$ are bireflection groups. There are exactly $71 \mathbb{Q}$-classes that do not consist of bireflection groups. By the foregoing, they lead to non-Cohen-Macaulay multiplicative invariant algebras. The $\mathbb{Q}$-classes consisting of bireflection groups have not been systematically investigated yet. The searches in rank 4 were done with [GAP].

\section{ACKNOWLEDGMENTS}

Some of the research for this article was carried out during a workshop in Seattle (August 2003) funded by Leverhulme Research Interchange Grant F/00158/X and during the symposium "Ring Theory" in Warwick, UK (September 2003). The results described here were reported in the special session "Algebras and Their Representations" at the AMS-meeting in Chapel Hill (October 2003). Many thanks to Bob Guralnick for his helpful comments on an earlier version of this article.

\section{REFERENCES}

[Bou 1 N. Bourbaki, Groupes et algèbres de Lie, chapitres 4, 5 et 6, Hermann, Paris, 1968. MR0240238 (39:1590)

[Bou 2$]$, Algèbre commutative, chap. 5/6, Hermann, Paris, 1964. MR0194450 (33:2660) 
[Bou $]$, Algèbre commutative, chapitres 8 et 9, Masson, Paris, 1983. MR0722608 (86j:13001)

[Br] K.S. Brown, Cohomology of Groups, Springer-Verlag, New York, 1982. MR0672956 $(83 \mathrm{k}: 20002)$

[BH] W. Bruns and J. Herzog, Cohen-Macaulay Rings, Cambridge University Press, Cambridge, 1993. MR:1251956 (95h:13020)

[CR] C.W. Curtis and I. Reiner, Methods of Representation Theory, Volume 1, John Wiley \& Sons, New York, 1981. MR0632548 (82i:20001)

[GAP] The GAP Group, GAP - Groups, Algorithms, and Programming, Version 4.4; 2004, (http://www.gap-system.org).

$\left[\mathrm{G}_{1}\right] \quad$ N.L. Gordeev, Invariants of linear groups generated by matrices with two nonidentity eigenvalues (Russian), Zap. Nauchn. Sem. Leningrad. Otdel. Mat. Inst. Steklov. (LOMI) 114 (1982), 120-130. MR0669563 (83m:20067)

$\left[\mathrm{G}_{2}\right] \quad$ N.L. Gordeev, Finite linear groups whose algebras of invariants are complete intersections, Math. USSR Izv. 28 (1987), 335-379. MR0842586 (88a:13020)

[GK] N.L. Gordeev and G. Kemper, On the branch locus of quotients by finite groups and the depth of the algebra of invariants, J. Algebra 268 (2003), 22-38. MR2004478 (2004h:13010)

[GuS] R. Guralnick and J. Saxl, Generation of finite almost simple groups by conjugates, J. Algebra 268(2003), 519-571. MR.2009321

[HE] M. Hochster and J.A. Eagon, Cohen-Macaulay rings, invariant theory, and the generic perfection of determinantal loci, Amer. J. Math. 93 (1971), 1020-1058. MR0302643 $(46: 1787)$

[Hu] C. Huffman, Linear Groups containing an element with an eigenspace of codimension two, J. Algebra 34(1975), 260-287. MR0401936 (53:5762)

$[\mathrm{HuW}]$ C. Huffman and D. Wales, Linear groups containing an element with an eigenspace of codimension two, in: Proceedings of the Conference on Finite Groups, Univ. Utah, Park City, Utah, 1975, Academic Press, New York, 1976, pp. 425-429. MR0435239 (55:8199)

[I] I.M. Isaacs, Character Theory of Finite Groups, Academic Press, New York, 1976. MR 0460423 (57:417)

$[\mathrm{KW}] \mathrm{V}$. Kac and K. Watanabe, Finite linear groups whose ring of invariants is a complete intersection, Bull. Amer. Math. Soc. 6 (1982), 221-223. MR0640951 (83h:14042)

$\left[\mathrm{K}_{1}\right] \quad$ G. Kemper, Die Cohen-Macaulay-Eigenschaft in der modularen Invariantentheorie, Habilitationsschrift, Universität Heidelberg, 1999.

$\left[\mathrm{K}_{2}\right] \quad$ G. Kemper, The depth of invariant rings and cohomology (with an appendix by Kay Magaard), J. Algebra 245(2001), 463-531. MR1863889(2002h:13009)

$\left[\mathrm{L}_{1}\right] \quad$ M. Lorenz, Multiplicative Invariant Theory, Encyclopaedia of Mathematical Sciences, Vol. 135, Springer-Verlag, New York, 2005.

$\left[\mathrm{L}_{2}\right] \quad$ M. Lorenz, Multiplicative invariants and semigroup algebras, Algebras and Rep. Thy. 4 (2001), 293-304. MR.1852001 (2002i:13003)

[LPk] M. Lorenz and J. Pathak, On Cohen-Macaulay rings of invariants, J. Algebra 245 (2001), 247-264. MR:1868191 (2002h:13010)

[N] H. Nakajima, Quotient singularities which are complete intersections, Manuscripta Math. 48 (1984), 163-187. MR0753729 (86h:14039)

[Pk] J. Pathak, The Cohen-Macaulay property of multiplicative invariants, Ph.D. thesis, Temple University, 2003.

[S] L. Smith, Polynomial Invariants of Finite Groups, A. K. Peters, Wellesley, 1995. MR:1328644 (96f:13008)

[St] R. Steinberg, Differential equations invariant under finite reflection groups, Trans. Amer. Math. Soc. 112 (1964), 392-400. MR0167535 (29:4807)

[W] D. Wales, Linear groups of degree $n$ containing an involution with two eigenvalues -1 , J. Algebra 53 (1978), 58-67. MR0480770 (58:921)

[Wo] J.A. Wolf, Spaces of Constant Curvature, 5th ed., Publish or Perish, Inc., Wilmington, 1984. MR0928600 (88k:53002)

Department of Mathematics, Temple University, Philadelphia, Pennsylvania 19122

E-mail address: lorenz@math.temple.edu 